\title{
Comparison of range-of-motion and variability in upper body movements between transradial prosthesis users and able-bodied controls when executing goal-oriented tasks
}

\author{
Matthew J Major ${ }^{1,2^{*}}$, Rebecca L Stine ${ }^{1,2}$, Craig W Heckathorne ${ }^{1}$, Stefania Fatone ${ }^{1}$ and Steven A Gard ${ }^{1,2}$
}

\begin{abstract}
Background: Current upper limb prostheses do not replace the active degrees-of-freedom distal to the elbow inherent to intact physiology. Limited evidence suggests that transradial prosthesis users demonstrate shoulder and trunk movements to compensate for these missing volitional degrees-of-freedom. The purpose of this study was to enhance understanding of the effects of prosthesis use on motor performance by comparing the movement quality of upper body kinematics between transradial prosthesis users and able-bodied controls when executing goal-oriented tasks that reflect activities of daily living.

Methods: Upper body kinematics were collected on six able-bodied controls and seven myoelectric transradial prosthesis users during execution of goal-oriented tasks. Range-of-motion, absolute kinematic variability (standard deviation), and kinematic repeatability (adjusted coefficient-of-multiple-determination) were quantified for trunk motion in three planes, shoulder flexion/extension, shoulder ab/adduction, and elbow flexion/extension across five trials per task. Linear mixed models analysis assessed between-group differences and correlation analysis evaluated association between prosthesis experience and kinematic repeatability.
\end{abstract}

Results: Across tasks, prosthesis users demonstrated increased trunk motion in all three planes and shoulder abduction compared to controls ( $p \leq 0.004$ ). Absolute kinematic variability was greater for prosthesis users for all degrees-of-freedom irrespective of task, but was significant only for degrees-of-freedom that demonstrated increased range-of-motion ( $p \leq 0.003$ ). For degrees-of-freedom that did not display increased absolute variability for prosthesis users, able-bodied kinematics were characterized by significantly greater repeatability $(p \leq 0.015)$. Prosthesis experience had a strong positive relationship with average kinematic repeatability $(r=0.790, p=0.034)$.

Conclusions: The use of shoulder and trunk movements by prosthesis users as compensatory motions to execute goal-oriented tasks demonstrates the flexibility and adaptability of the motor system. Increased variability in movement suggests that prosthesis users do not converge on a defined motor strategy to the same degree as able-bodied individuals. Kinematic repeatability may increase with prosthesis experience, or encourage continued device use, and future work is warranted to explore these relationships. As compensatory dynamics may be necessary to improve functionality of transradial prostheses, users may benefit from dedicated training that encourages optimization of these dynamics to facilitate execution of daily living activity, and fosters adaptable but reliable motor strategies.

Keywords: Upper limb, Transradial amputation, Kinematics, Trunk, Prosthesis

\footnotetext{
* Correspondence: matthew-major@northwestern.edu

${ }^{1}$ Northwestern University Prosthetics-Orthotics Center, Northwestern University

Feinberg School of Medicine, 680 N Lake Shore Dr, Suite 1100, Chicago, IL

60611, USA

${ }^{2}$ Jesse Brown VA Medical Center, 820 S Damen Ave, Chicago, IL 60612, USA
} 


\section{Background}

The musculoskeletal architecture of the upper body (i.e., trunk and arms) contains redundant degrees-of-freedom (DoFs) from which the central nervous system (CNS) has the benefit of selecting various task-equivalent motor strategies and associated inter-joint coordination patterns to satisfy task requirements [1-3]. The advantage of this redundancy is that the neuromuscular system is highly adaptable and capable of accounting for a loss in DoFs due to pathology [4-9]. This motor adaptability is of critical advantage for individuals with transradial limb loss in the context of task performance, as typical upper limb prostheses do not replace the active DoFs distal to the elbow inherent to intact physiology, including forearm pronation/supination and wrist flexion/extension [10]. There is substantial evidence to suggest that due to loss of active DoFs, users of transradial prostheses employ compensatory movements predominantly at the trunk and shoulder to execute goal-oriented tasks [5,6]. Studies have further emphasized the impact of forearm and wrist movement on compensatory motions during execution of goaloriented tasks by demonstrating that able-bodied individuals exhibit similar compensatory motions as transradial prosthesis users (e.g., lateral trunk flexion) when forearm and wrist movement is restricted [11,12], and fixing the prosthetic wrist in different flexion angles affects prosthetic arm compensatory movements (e.g., shoulder anteversion and abduction) [13].

Despite indication that compensatory motions may result in overuse injuries [12,14], the opinion exists that this behavior should not be discouraged when operating an upper limb prosthesis as it demonstrates adaptability given the functional limitations of the device, and appropriate strategies should be identified to facilitate task execution [15]. The key to control when using a transradial prosthesis is to properly manage the remaining DoFs [5], and studies indicate that the CNSs of prosthesis users create accurate internal models of the affected arm through residual sensory feedback and utilize adapted motor control strategies (i.e., coordinated joint torques and geometry) for task execution [16-19]. Importantly, evidence suggests that although upper limb prosthesis users adapt to novel task environments, they demonstrate increased motor variability, but dedicated experience and training with the device may facilitate long-term motor adaptation and increased kinematic repeatability $[18,20,21]$. Therefore, the ultimate goal of rehabilitation therapy to enhance motor learning and refine these movements through practice via task repetition is reasonable [18-20,22,23]. A certain level and type of kinematic variability may be considered an asset to upper limb prosthesis users for the purpose of exploiting motor flexibility that may aid task execution [24], but the amount of variability associated with upper body movement in this group compared to able-bodied controls has not been adequately explored. Furthermore, dependable motor behavior, as reflected by reduced movement variability that results from appropriate prosthetic training, may reflect better prosthetic control, which could prevent user dissatisfaction and device abandonment [25-27]. However, despite the common therapeutic goal of enhancing movement quality, there still remains a dearth of information on the characteristics of upper body compensatory movements and associated variability of transradial prosthesis users when executing goal-oriented tasks, and the relationship of these dynamics with device experience [20]. This is partially due to the paucity of upper limb prosthetic research studies incorporating methods for the characterization of upper body kinematics as a form of outcome measurement to assess performance $[15,20]$.

The objectives of this study were to compare upper body movements and their associated variability between able-bodied individuals and transradial prosthesis users during execution of goal-oriented tasks that reflect activities of daily living (ADLs), and to assess relationships between movement variability and prosthesis experience. We hypothesize that: 1) prosthesis users will display altered range-of-motion (RoM) at the trunk and shoulder compared to able-bodied individuals to compensate for a loss in active distal DoFs, 2) prosthesis users will display increased within-subject movement variability as they recruit compensatory motions to execute novel goaloriented tasks, and 3) movement repeatability of prosthesis users has a positive direct relationship with prosthesis experience. Importantly, upper limb prosthesis use performance has traditionally been assessed through the use of clinical outcome measures [28-30]. Although these measures provide useful clinically-relevant information, they only provide information on functional outcome and are unable to assess the contribution of movement quality to overall performance [23]. The results from this study will allow for improved insight into the movement quality associated with upper limb prosthesis user performance, contribute to quantification of compensatory movements when executing goal-oriented tasks, and enhance understanding of how the motor strategies of prosthesis users differ from able-bodied individuals and are related to device experience.

\section{Methods}

\section{Participants}

Participant inclusion criteria required that they be free of neurological and musculoskeletal pathology (apart from upper limb loss) that would impair upper limb motor control during goal-oriented task execution in a seated position. Participants with limb loss were required to have unilateral loss at the transradial level and currently operate a prosthesis with myoelectric control that would be used during the experiments. A requirement was 
that the prosthesis be self-suspending with a supracondylar socket design. Ethical approval was obtained from the University Institutional Review Board, and informed consent was provided by each participant prior to data collection.

Six able-bodied individuals (3 male, 3 female, $35 \pm$ 11 years) and seven individuals with limb loss at the transradial level (5 male, 2 female, $49 \pm 18$ years) participated in this study. Limb loss etiology included traumatic $(n=4)$ and congenital $(n=3)$. User devices included the System Electric Hand $(\mathrm{n}=1$; Otto Bock, Duderstadt, Germany), MyoHand VariPlus Speed Hand ( $\mathrm{n}=1$; Otto Bock), Transcarpal Hand ( $\mathrm{n}=1$; Otto Bock), Motion Control Hand ( $\mathrm{n}=1$; Motion Control, Salt Lake City, UT), $\mathrm{i}$-Limb Ultra Revolution ( $\mathrm{n}=1$; Touch Bionics, Livingston, UK), i-Limb Ultra ( $\mathrm{n}=1$; Touch Bionics), and i-Limb Hand ( $\mathrm{n}=1$; Touch Bionics). For all participants with traumatic amputation, the amputated limb was considered their dominant limb prior to the amputation and the average time since amputation was $9.5 \pm 11.0$ years. Across all subjects, the average reported prosthesis experience was $20.3 \pm 18.1$ years. Prosthetic socket comfort was quantified with the socket comfort score [31], which asks users to rate their prosthesis on an ordinal scale from 0 (most uncomfortable socket imaginable) to 10 (most comfortable socket imaginable), and the average score was $8.9 \pm 1.1$, suggesting a high level of comfort across participants.

\section{Experimental protocol}

Participants were requested to execute five goal-oriented tasks while seated at a table of standard height (73.7 and $28 \mathrm{~cm}$ from floor and seat top, respectively). The tasks and associated protocol were adopted from the Southampton Hand Assessment Procedure (SHAP) [32], as this assessment provided a standardized protocol for controlled, repeated performance of goal-oriented tasks that reflect ADLs:

1) Food cutting - picking up and positioning a knife to slice through a small plasticine roll located at midline of the body.

2) Page turning - grasping a page, located on one side of the body, with the hand on the contralateral side and turning the page over to the ipsilateral side as if turning the page of a book.

3) Carton pouring - lifting a carton, located at midline of the body, and emptying the liquid contents into a jar on the contralateral side with minimal spilling.

4) Lifting and transferring a weighted object - lifting a liquid-filled jar, located on one side of the body, with the hand on the contralateral side and transferring to the ipsilateral side over a low-level barrier at midline of the body.
5) Lifting and transferring a tray - lifting a tray, located on the contralateral side of the body, with both hands and transferring to the ipsilateral side over a low-level barrier at midline of the body.

Per the SHAP protocol, participants were requested to complete each task as fast as possible. Tasks did not require participants to position their joints/segments in any particular orientation in space, but only to complete the task requirements. Able-bodied controls and prosthesis users performed these tasks using their nondominant and prosthetic limb, respectively, for unimanual tasks (page turning, carton pouring, lifting and transferring a weighted object). For the tasks of page turning and lifting and transferring a weighted object and tray, the object was first positioned on the side of the body opposite to the non-dominant or prosthetic limb. Prior to data collection and the start of each task, the participants rested both hands on the table in the same position (Figure 1a). Once the participants were requested to start the task, they struck a large button at midline of the body with their non-dominant or prosthetic limb (Figure 1b) and this event denoted the start of the task. Following execution of each task, the participant again struck the button and this denoted the end of the task. Five trials of each task were performed in random order to minimize acute learning effects [22], as the intent was to observe performance of the desired tasks and not capture changes due to learning through repetition [23]. Participants were permitted practice to familiarize themselves with the task requirements prior to data collection.

\section{Data collection and analysis}

Kinematic data were collected with a digital motion capture system (Motion Analysis Corporation, Santa Rosa, CA) at $120 \mathrm{~Hz}$ using a custom upper extremity retroreflective marker set to define three rigid-body segments (forearm, upper arm, and trunk) and three additional markers on the table surface to define the work plane. A subset of the anatomical locations of retro-reflective

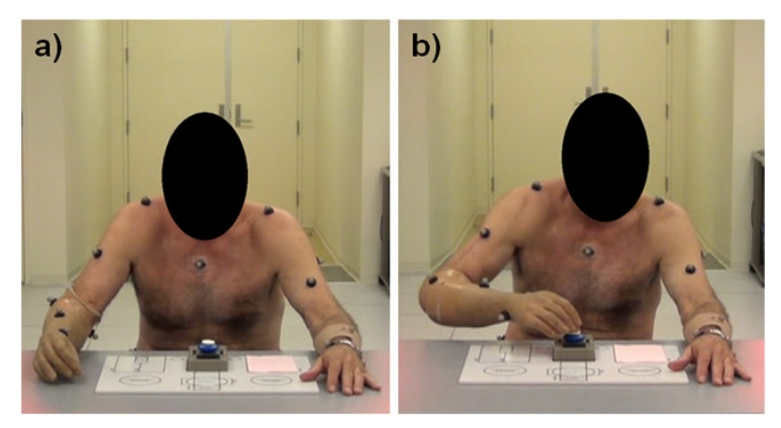

Figure 1 Image of a prosthesis user in the position prior to the start of task (a), and at the start and end of task execution (b). 
markers used in this analysis include: C7 spinous process, sacrum, top of the sternum, bilateral acromion processes, medial and lateral humeral epicondyles, and radial and ulnar styloids. For prosthesis users, the locations of the epicondyle and styloid markers mimicked the relative position of these bony landmarks on the intact arm. Virtual elbow and wrist joint centers were created as the midpoint between the epicondyle and styloid markers, respectively. The forearm was defined as the segment linking the virtual wrist and elbow centers, and the upper arm was defined as the segment linking the virtual elbow center and acromion process marker.

Upper body kinematics (i.e., shoulder flexion/extension, shoulder ab/adduction, elbow flexion/extension, trunk lateral flexion, trunk transverse rotation, and trunk forward flexion) were estimated from the five trials of each task using custom software (Matlab, Natick, MA). A trunk-based local reference frame was calculated using the C7, sacrum, and sternum markers to define the anatomical sagittal, frontal, and transverse planes. Shoulder flexion/extension was defined as the angle between the upper arm and trunk anterior-posterior axis (zero angular displacement defined as perpendicular alignment of the upper arm and trunk axis). Shoulder abduction was defined as the angle between the upper arm and shoulder axis created by the acromion markers (zero angular displacement defined as perpendicular alignment of the upper arm and shoulder axis). Elbow flexion/extension was defined as the angle between the upper arm and forearm $\left(180^{\circ}\right.$ of angular displacement was defined as full elbow extension). Trunk lateral flexion was defined as the angle between the trunk inferior-superior axis and table medial-lateral axis (i.e., table width) projected onto the frontal plane (zero angular displacement defined as perpendicular alignment of the trunk and table axes). Trunk transverse rotation was defined as the angle between the shoulder axis and table medial-lateral axis projected onto the transverse plane (zero angular displacement defined as parallel alignment of the shoulder and table axes). Trunk forward flexion was defined as the angle between the trunk inferior-superior axis and table anterior-posterior axis projected onto the sagittal plane (zero angular displacement defined as perpendicular alignment of the trunk and table axes). Kinematic data were filtered with a bidirectional 4th order low-pass Butterworth filter at $6 \mathrm{~Hz}$. DoF angle trajectories were normalized to task completion time (i.e., from the first to second button strike).

For each task, average RoM, absolute kinematic variability and kinematic repeatability of the observed DoF angles across five trials were calculated for each participant. The RoM of each DoF was estimated by subtracting the maximum and minimum recorded angles. Absolute kinematic variability across the five trials was estimated as the average standard deviation (SD) across all time points of the normalized DoF angle trajectory. Kinematic repeatability was estimated by the adjusted coefficient of multiple determination (CMD), also commonly reported as the $R^{2}$ value [33]. The CMD provides a statistical estimate of the similarity between waveforms and typically ranges between 0 and 1 [33], with values closer to 1 indicating increased repeatability. Although a useful method for assessing kinematic repeatability, limitations with the CMD method have been reported, such as a direct relationship with DoF RoM and lack of information on absolute measurement variability [34,35]. Consequently, $\mathrm{SD}$ is also reported here to complement CMD as a measure of absolute variability and between-group comparisons of CMD are limited to an analysis for each respective DoF $[34,35]$. In light of its utility, CMD has successfully been applied to upper body kinematic studies on children with cerebral palsy executing goal-oriented tasks [36-41].

\section{Statistical analysis}

Statistical analyses were conducted using SPSS (IBM, Armonk, NY) and the critical alpha was set at 0.05 . Normality of the data was confirmed using the Shapiro-Wilk test. A linear mixed model (LMM) analysis (fixed factor: group, task, group $\times$ task; random factor: subject) was used to assess if group classification resulted in individual DoF RoM, SD, and CMD differences. Group means and 95\% confidence intervals were calculated for each task to illustrate task-specific differences and group $\times$ task interaction effects. An overall CMD value was calculated for each participant by averaging CMD values across DoFs and tasks (as a measure of overall withinsubject kinematic repeatability) and a Pearson correlation coefficient was estimated between prosthesis users' overall CMD values and prosthesis experience.

\section{Results}

The majority of prosthesis users were unable to routinely execute tasks 1 (food cutting) and 2 (page turning) as required by the protocol due to their inability to maintain grip of the knife and fully rotate the page from front to back, respectively, with their prostheses. Consequently, data from these tasks were eliminated from the analysis. Additionally, one prosthesis user was unable to execute task 5 (tray lift and transfer) due to inadvertent opening of the prosthetic hand and these data are missing from the analysis. To aid in visualization of kinematic results, an example of group ensemble average kinematics of task 3 (carton pouring) is presented in Figure 2. Group data for RoM, SD, and CMD of tasks 3, 4, and 5 are displayed in Figures 3, 4, and 5, respectively, and the LMM statistical results are displayed in Table 1.

Results indicate that prosthesis users demonstrate a significant increase in shoulder abduction, trunk 

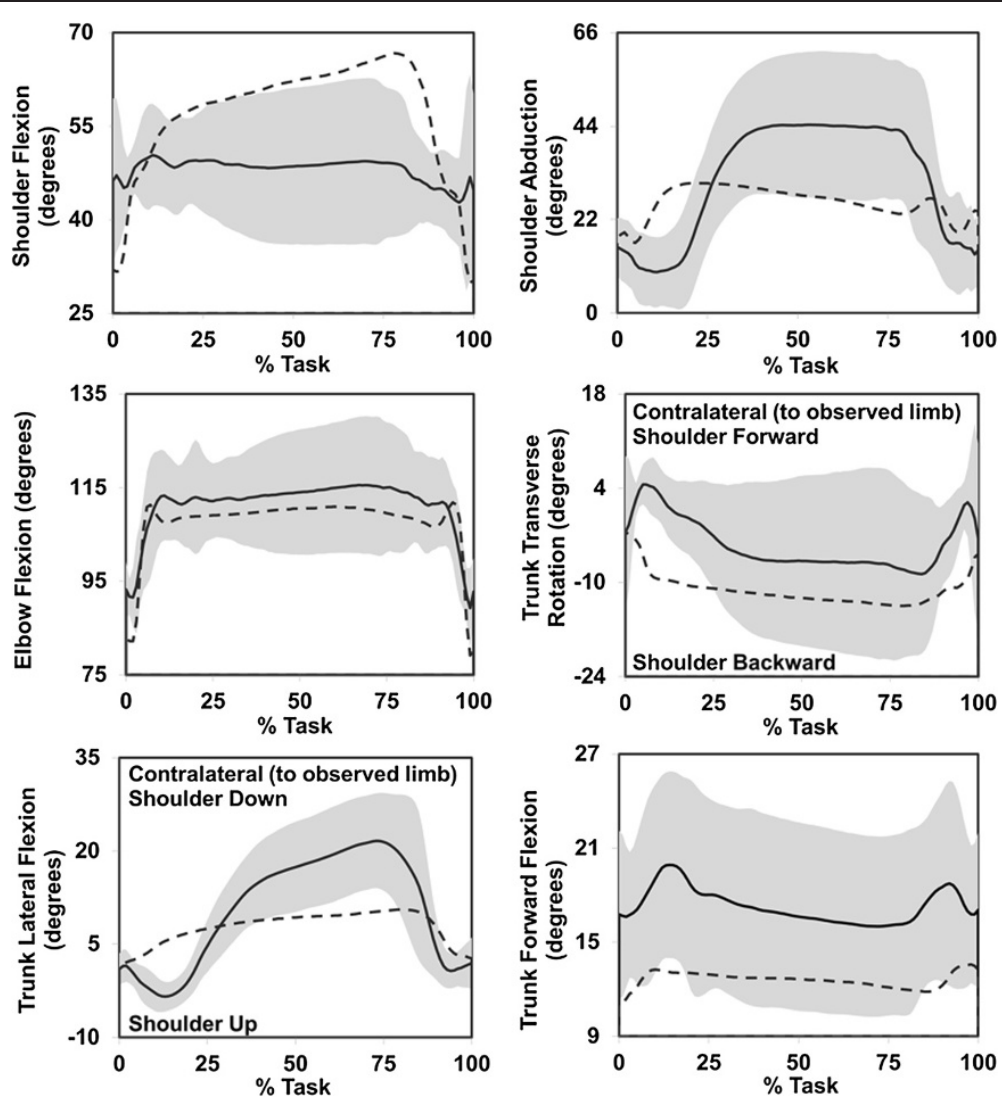

Figure 2 Group ensemble average kinematic profiles of able-bodied (average = dashed line) and prosthesis users (average = solid line; standard deviation = shaded band) executing task 3 (carton pouring). Neutral for all DoF angles is $0^{\circ}$, apart from elbow flexion where full extension is $180^{\circ}$ and smaller values denote elbow flexion.

transverse rotation, trunk lateral flexion, and trunk forward flexion RoM. Additionally, for prosthesis users, all of the DoFs with increased RoM apart from trunk forward flexion also displayed increased absolute kinematic variability as reflected by increased average SD. For shoulder flexion/extension, elbow flexion/extension, and trunk forward flexion, which did not display differences in absolute kinematic variability (SD), able-bodied controls demonstrated increased kinematic repeatability as reflected by greater CMD values. Few interaction effects (group $\times$ task) were revealed by the statistical analyses, and these were primarily limited to RoM of shoulder flexion/extension and abduction, and trunk lateral and forward flexion (see Figures 3, 4, and 5), demonstrating that these DoFs RoM varied between groups based on task. Overall CMD values of the prosthesis users were strongly correlated with prosthesis experience in years $(\mathrm{r}=0.790, \mathrm{p}=0.034)$ with an average of $0.67 \pm 0.07$ (range: 0.54 to 0.74 ), while the average able-bodied CMD value was $0.71 \pm 0.03$ (range: 0.67 to 0.74 ).

\section{Discussion}

Controlled goal-oriented tasks that reflected ADLs were executed by prosthesis users and able-bodied controls to determine if: 1) the loss of active distal DoFs associated with a transradial prosthesis encouraged alterations in kinematic RoM, 2) transradial prosthesis users demonstrated increased kinematic variability, and 3) prosthesis experience was associated with increased kinematic repeatability.

\section{Degrees-of-freedom range-of-motion}

Prosthesis users demonstrated greater shoulder abduction and trunk RoM than the able-bodied controls when observed across tasks (Figure 3). Generally, the kinematic profiles of prosthesis users were distinct from those of able-bodied individuals, and Figure 2 provides an example of the unique management of upper body DoFs for prosthesis users. The use of available trunk DoFs to compensate for loss of active distal arm DoFs of transradial prosthesis users has been reported previously $[5,6]$ and these results agree with the literature. Given that the ability to manipulate end point position in the sagittal plane is 


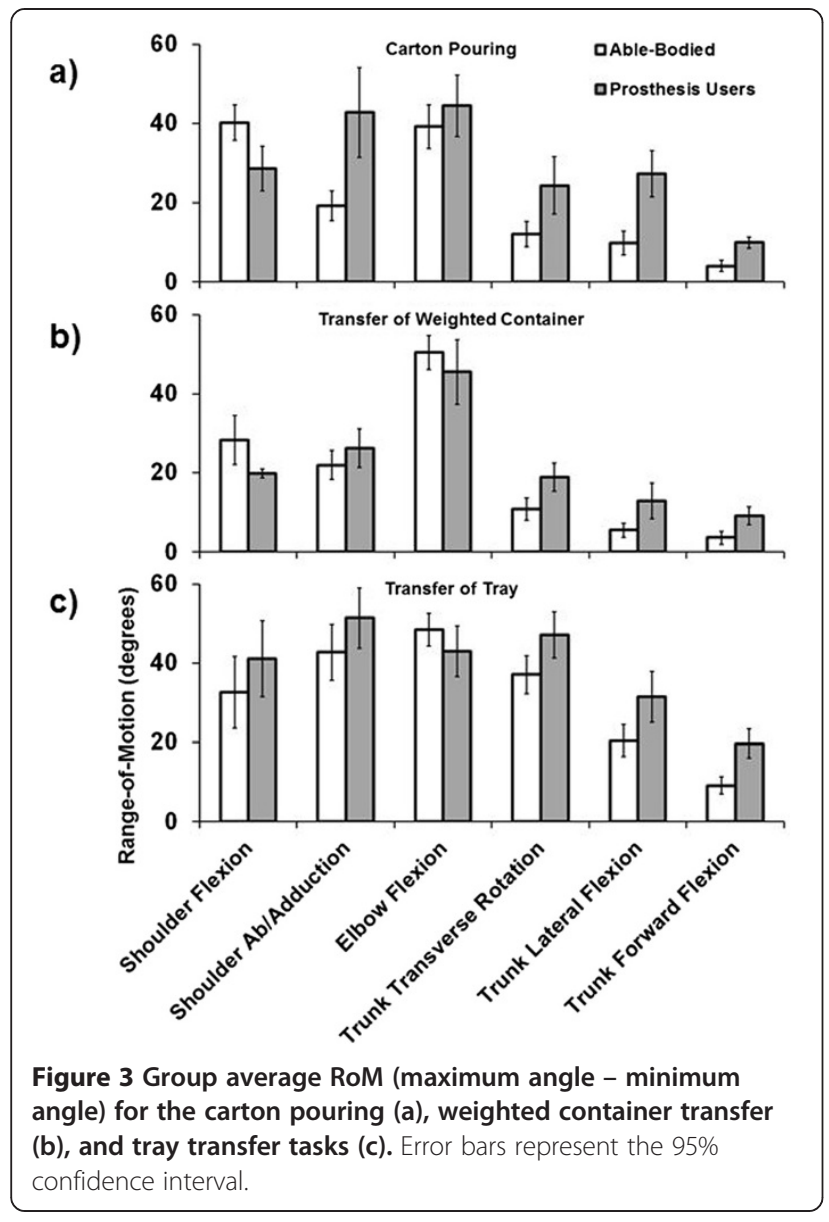

not affected by use of a transradial prosthesis, the absence of significant differences in shoulder and elbow flexion/extension RoM seems reasonable. Similar to individuals post-stroke and with frozen shoulder, transradial prosthesis users appear to be using the trunk as part of the kinematic chain to manipulate endpoint position [4-8]. The combination of increased shoulder abduction and trunk motion facilitates execution of the required tasks for prosthesis users, as these DoFs working in synergy would theoretically compensate for absence of active supination/ pronation of the forearm. Although there is some evidence to suggest that trunk restraint training reduces trunk compensatory motions and elicits modifications in arm kinematics to facilitate task execution in individuals poststroke [42-44], the complete loss of active distal DoFs in transradial prosthesis users likely requires consistent recruitment of the trunk DoFs. Given that transradial prosthesis users possess an internal model of the affected limb to drive motor patterns that are comparable to normal physiologic function [16-19], this further suggests that trunk and shoulder compensatory motions are essential voluntary behavior to facilitate task execution.

Overall, these results seem to support the idea that it may be advantageous to encourage compensatory movements a)

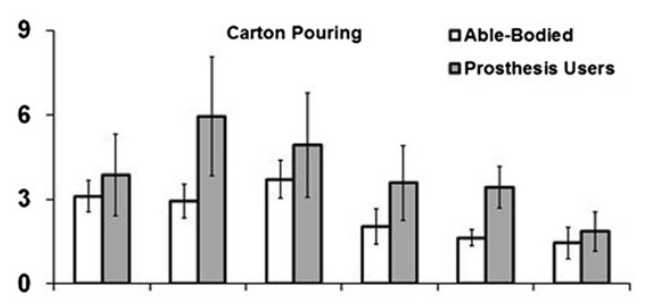

b)

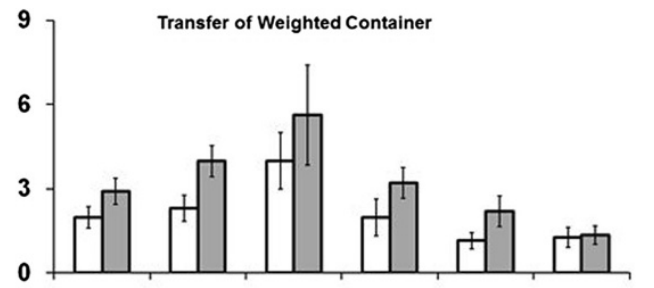

c)

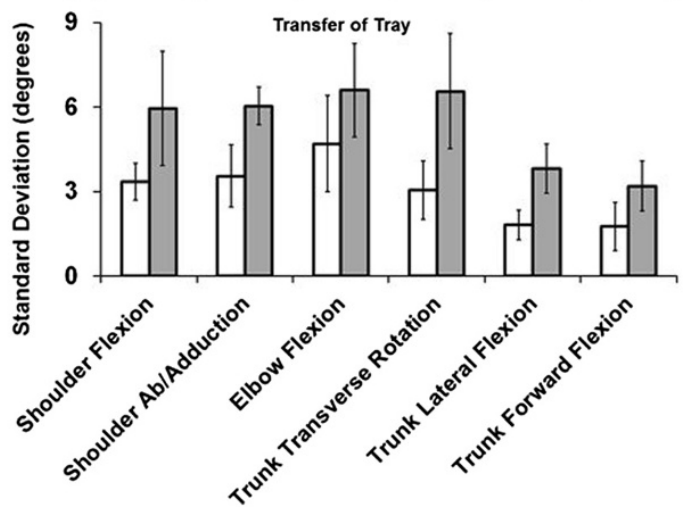

Figure 4 Group average SD for the carton pouring (a), weighted container transfer (b), and tray transfer tasks (c). Error bars represent the $95 \%$ confidence interval.

in a manner that is optimally ergonomic to facilitate task execution and minimize injury, and this may have implications for prosthetic training. The recruitment of trunk and shoulder motion demonstrates the flexibility of the upper body motor system and the adaptability of transradial prosthesis users when executing ADLs. Training that encourages successful management of the DoFs of the upper body could counter the functional limitations of currently available transradial prostheses, and although compensatory movements may impose unfavorable metabolic costs, the functional improvements may ultimately enhance user perception of device utility [25-27]. However, as there is indication that sustained trunk forward flexion compared to upright posture [45] and iso-directional coupled arm movement compared to anti-directional coupling [46] during standing increases metabolic cost, future research should investigate the impact of upper body compensatory motions and posture on metabolic cost during upper body goal-oriented task execution. Furthermore, with advances in upper limb prosthetic control techniques for active wrist rotation [47], the contribution of this voluntary DoF to upper body compensatory movements warrants investigation. 


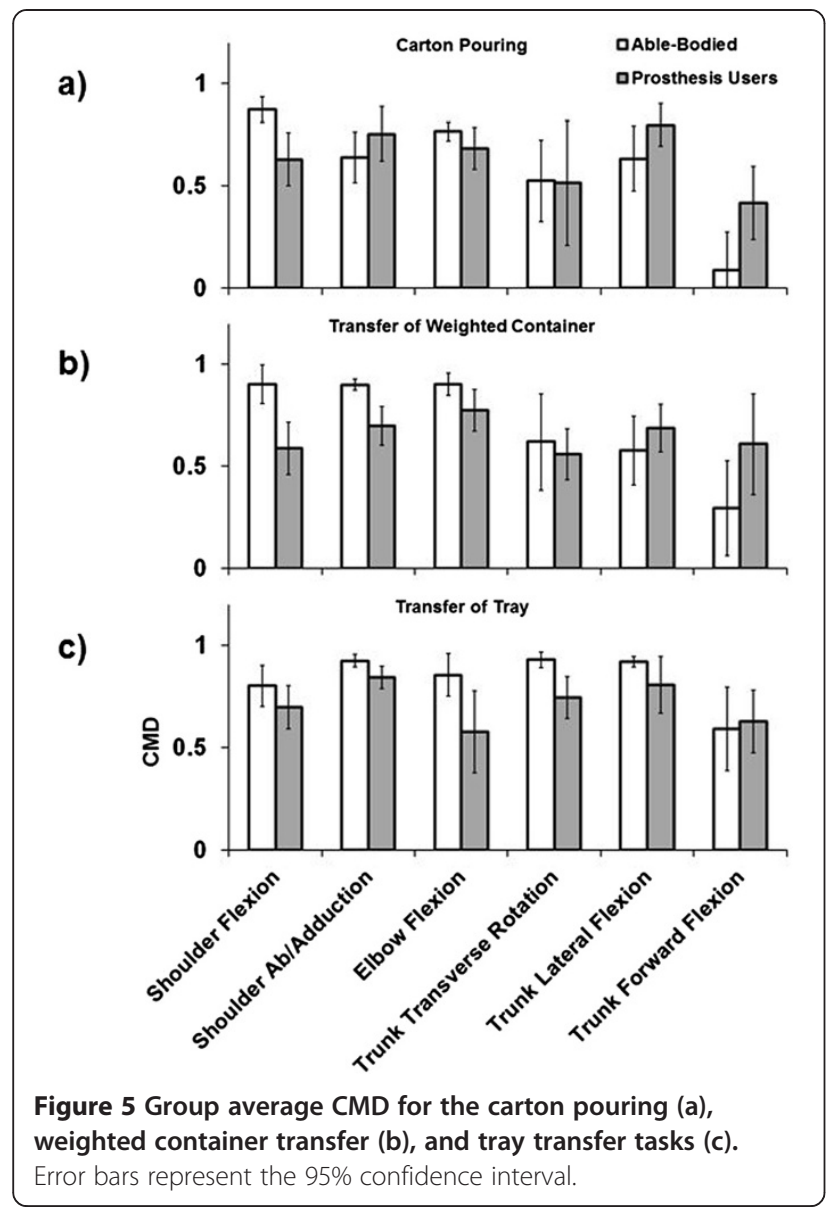

Kinematic variability

Apart from trunk forward flexion, the DoFs that displayed increased RoM in prosthesis users also demonstrated a significant increase in within-subject SD compared to able-bodied controls. These results suggest that these compensatory dynamics appear to be associated with an increase in average variability of movement, such that prosthesis users generally do not converge on a defined motor synergy to the degree observed in able-bodied individuals. The DoFs that did not demonstrate a significant increase in SD for prosthesis users, which would otherwise indicate greater variability, displayed a significant increase in CMD for able-bodied controls. This result suggests greater kinematic repeatability of controls compared to prosthesis users for those DoFs, and also the complementary information provided by the SD and CMD techniques. The increased shoulder and trunk variability of prosthesis users did not preclude execution of tasks 3, 4, and 5, and this further demonstrates the utility of the motor flexibility associated with the upper body kinematic chain to manipulate end point position. Consequently, this variability may be seen as advantageous when operating a transradial prosthesis with reduced active distal DoFs and reflective of healthy motor performance $[24,48]$, where the various DoFs are able to compensate for each other to ensure repeated task execution. However, this motor flexibility is not without limitation, as the majority of participants were unable to rotate a page 180 degrees as required by page turning in a book (task 2).

Increased variability may also be viewed as diminished movement quality when compared to able-bodied individuals, as real-time adaptation during execution was not necessary since there were no external factors such as perturbation. Although the tasks executed in this study did not require specific joint/segment configurations, able-bodied individuals were able to maintain relatively low levels of within-subject absolute variability (Figure 5) and while only significant for some DoFs, prosthesis user variability averages were consistently greater across all tasks and DoFs. Increased movement variability may consequently be identified by prosthesis users as inconsistent or unreliable device response, and hence increase end-user frustration and diminish the perceived utility of the device as has been demonstrated during human interaction with computers via a controller $[49,50]$. Frustration may be a realistic outcome if prosthesis users perceive kinematic variability as an obstacle to consistent goal attainment, and anecdotally, such a response was observed in this study when some users were unable to reposition the knife in their prosthesis to replicate execution of task 1.

Overall kinematic repeatability shared a direct relationship with prosthesis experience. As only prosthesis users were tested in this study and only during one testing session, the causal relationship between experience and variability is unknown, i.e., whether kinematic variability decreases over time with device experience, or low levels of variability encourage continued device use. However, motor adaptation and learning through practice and experience is essential for upper extremity neurorehabilitation to minimize variable outcomes and maximize likelihood of successful task execution [23]. Similarly, there is some evidence to suggest that an inverse relationship exists between athletic skill and movement variability for those dynamics responsible for performance [51-53]. Consequently, it may be reasonable to postulate that kinematic variability of goal-oriented tasks would decrease with training and experience, such that those individuals with greater experience would possess an enhanced ability to integrate various forms of sensory feedback to execute tasks with near normal reliability $[20,54]$. In fact, the linear trend in experience and kinematic repeatability was such that as experience increased, repeatability approached values equivalent to that of able-bodied controls. These results further encourage investigations into the relationships between kinematic variability, device experience, training modalities, and user satisfaction. 
Table 1 Linear mixed model results for group and group $\times$ task interaction effects for each DoF

\begin{tabular}{|c|c|c|c|c|c|}
\hline \multirow[b]{2}{*}{ Degrees-of-freedom } & \multirow[b]{2}{*}{ Parameter } & \multicolumn{2}{|c|}{ Group effect } & \multicolumn{2}{|c|}{ Group $\times$ Task interaction effect } \\
\hline & & F-value & p-value & F-value & p-value \\
\hline & RoM & 1.468 & 0.251 & $6.613^{*}$ & $0.006^{*}$ \\
\hline \multirow[t]{3}{*}{ Shoulder flexion/extension } & SD & 4.739 & 0.052 & $3.580^{*}$ & $0.046^{*}$ \\
\hline & CMD & $15.714^{*}$ & $0.002^{*}$ & 2.195 & 0.136 \\
\hline & RoM & $13.268^{*}$ & $0.004^{*}$ & $4.424^{*}$ & $0.024^{*}$ \\
\hline \multirow[t]{3}{*}{ Shoulder ab/adduction } & SD & $16.658^{*}$ & $0.002^{*}$ & 0.921 & 0.413 \\
\hline & CMD & 1.048 & 0.328 & $9.782^{*}$ & $0.001^{*}$ \\
\hline & RoM & 0.092 & 0.767 & 3.067 & 0.068 \\
\hline \multirow[t]{3}{*}{ Elbow flexion/extension } & SD & 3.568 & 0.085 & 0.117 & 0.890 \\
\hline & CMD & $8.341^{*}$ & $0.015^{*}$ & 1.813 & 0.187 \\
\hline & RoM & $23.133^{*}$ & $0.001^{*}$ & 0.359 & 0.702 \\
\hline \multirow[t]{3}{*}{ Trunk transverse rotation } & SD & $14.062^{*}$ & $0.003^{*}$ & 2.470 & 0.108 \\
\hline & CMD & 1.058 & 0.325 & 0.386 & 0.684 \\
\hline & RoM & $21.476^{*}$ & $0.001^{*}$ & $3.653^{*}$ & $0.044^{*}$ \\
\hline \multirow[t]{3}{*}{ Trunk lateral flexion } & SD & $35.563^{*}$ & $<0.001^{*}$ & 1.393 & 0.271 \\
\hline & CMD & 0.901 & 0.362 & 2.737 & 0.087 \\
\hline & RoM & $40.988^{*}$ & $<0.001^{*}$ & $4.263^{*}$ & $0.028^{*}$ \\
\hline \multirow[t]{2}{*}{ Trunk forward flexion } & SD & 4.030 & 0.700 & 2.670 & 0.092 \\
\hline & $\mathrm{CMD}$ & $7.040^{*}$ & $0.012^{*}$ & 1.204 & 0.313 \\
\hline
\end{tabular}

Significant effects $(p \leq 0.05)$ are denoted by an asterisk.

A limitation of this study is that due to the small sample size, which reflects the practicalities of in-vivo prosthetic upper limb research given a small overall population size, prosthetic designs varied between participants. Although there is limited evidence to suggest that there is no difference in functional performance between single DoF and multi-articulated prosthetic hands [55], the use of different devices may have introduced a confounding factor to kinematic performance. However, the mixed model analysis helped account for within-subject variability across tasks and thereby lends confidence that the statistical results reflect true differences between groups. Additionally, only a selection of tasks were executed by participants in this study and, although they reflect ADLs, generalization of the results should be made with caution based on this limitation. Finally, the assessments of kinematic variability to produce single statistical measures were based on time-normalized kinematic profiles that are unable to identify the individual contributions of phase and magnitude differences. Other, more sophisticated, methods to quantify these aspects of variability, such as dynamic time-warping [56], may offer additional insight into this outcome measure.

\section{Conclusions}

Transradial prosthesis users utilize shoulder abduction and trunk movement as compensatory motions to execute goal-oriented tasks, and the majority of these motions are accompanied by increased kinematic variability when compared to able-bodied controls. The average repeatability of upper body kinematics was positively associated with prosthesis experience. As these dynamics may be necessary to compensate for the absence of active distal DoFs in the prosthetic arm, transradial prosthesis users may benefit from dedicated training that: 1 ) encourages optimization of these dynamics to facilitate execution of ADLs, and 2) fosters adaptable but reliable motor strategies. Importantly, results from this study further emphasize the utility of motion capture for investigating movement quality of upper limb prosthesis users, which can complement information on functional clinical outcomes when assessing overall performance. As new upper limb prosthetic designs with increased active DoFs become commercially available, more sophisticated outcome metrics that consider functional clinical outcomes and movement quality may be necessary to effectively assess the contribution of these design features to user performance.

\section{Abbreviations}

DoF: Degree-of-freedom; CNS: Central nervous system; ADL: Activity of daily living; RoM: Range-of-motion; SHAP: Southampton Hand Assessment Procedure; SD: Standard deviation; CMD: Adjusted coefficient of multiple determination; LMM: Linear mixed model.

\section{Competing interests}

The authors declare no conflict of interest with this work. 


\section{Authors' contributions}

$\mathrm{RS}$ and $\mathrm{CH}$ carried out data collection. MM and RS carried out data processing. MM performed statistical analyses and drafted the manuscript. $\mathrm{CH}$ and $\mathrm{MM}$ conceived of the study, and $\mathrm{CH}, \mathrm{MM}$, and RS participated in its design. All authors participated in results interpretation, manuscript revisions, and read and approved the final manuscript.

\section{Acknowledgements}

The authors would like to thank Elisah Pietersma for her assistance with development of the analysis software, Dr. Laurence Kenney for his critical review of the manuscript, and Dr. Jody Ciolino of the Northwestern University Biostatistics Collaboration Center for statistics consultation. This work was supported by the National Institute on Disability and Rehabilitation Research (US Department of Education) under grant H133E080009, and the David Rubin, MD, Enrichment Fund. The opinions contained in this publication are those of the grantee and do not necessarily reflect those of the Department of Education.

Received: 20 May 2014 Accepted: 2 September 2014

Published: 6 September 2014

\section{References}

1. Bernstein NA: The co-ordination and regulation of movements. Oxford, UK: Pergamon Press; 1967.

2. Mussa Ivaldi FA, Morasso P, Zaccaria R: Kinematic networks. A distributed model for representing and regularizing motor redundancy. Biol Cybern 1988, 60:1-16.

3. Ma S, Feldman AG: Two functionally different synergies during arm reaching movements involving the trunk. J Neurophysiol 1995, 73:2120-2122

4. Cirstea MC, Levin MF: Compensatory strategies for reaching in stroke. Brain 2000, 123(Pt 5):940-953

5. Metzger AJ, Dromerick AW, Holley RJ, Lum PS: Characterization of compensatory trunk movements during prosthetic upper limb reaching tasks. Arch Phys Med Rehabil 2012, 93:2029-2034

6. Carey SL, Dubey RV, Bauer GS, Highsmith MJ: Kinematic comparison of myoelectric and body powered prostheses while performing common activities. Prosthet Orthot Int 2009, 33:179-186.

7. Robertson JV, Roby-Brami A: The trunk as a part of the kinematic chain for reaching movements in healthy subjects and hemiparetic patients. Brain Res 2011, 1382:137-146.

8. Fayad F, Hanneton S, Lefevre-Colau MM, Poiraudeau S, Revel M, Roby-Brami A: The trunk as a part of the kinematic chain for arm elevation in healthy subjects and in patients with frozen shoulder. Brain Res 2008, 1191:107-115.

9. Wee SK, Hughes AM, Warner M, Burridge JH: Trunk restraint to promote upper extremity recovery in stroke patients: a systematic review and meta-analysis. Neurorehabil Neural Repair 2014, 28(7):660-677.

10. III Meier RH, Atkins DJ: Functional restoration of adults and children with upper extremity amputation. New York, NY: Demos Medical Publishing, Inc:; 2004.

11. Carey SL, Jason Highsmith M, Maitland ME, Dubey RV: Compensatory movements of transradial prosthesis users during common tasks. Clin Biomech 2008, 23:1128-1135.

12. Mell AG, Childress BL, Hughes RE: The effect of wearing a wrist splint on shoulder kinematics during object manipulation. Arch Phys Med Rehabil 2005, 86:1661-1664

13. Bertels T, Schmalz T, Ludwigs E: Objectifying the functional advantages of prosthetic wrist flexion. J Prosthet Orthot 2009, 21:74-78.

14. Ostlie K, Franklin RJ, Skjeldal OH, Skrondal A, Magnus P: Musculoskeletal pain and overuse syndromes in adult acquired major upper-limb amputees. Arch Phys Med Rehabil 2011, 92:1967-1973 e1961.

15. Bouwsema H, Kyberd PJ, Hill W, van der Sluis CK, Bongers RM: Determining skill level in myoelectric prosthesis use with multiple outcome measures. J Rehabil Res Dev 2012, 49:1331-1348.

16. Metzger AJ, Dromerick AW, Schabowsky CN, Holley RJ, Monroe B, Lum PS: Feedforward control strategies of subjects with transradial amputation in planar reaching. J Rehabil Res Dev 2010, 47:201-211.

17. Dromerick AW, Schabowsky CN, Holley RJ, Monroe B, Markotic A, Lum PS: Effect of training on upper-extremity prosthetic performance and motor learning: a single-case study. Arch Phys Med Rehabil 2008, 89:1199-1204.
18. Schabowsky CN, Dromerick AW, Holley RJ, Monroe B, Lum PS: Trans-radial upper extremity amputees are capable of adapting to a novel dynamic environment. Exp Brain Res 2008, 188:589-601

19. Bouwsema $H$, van der Sluis CK, Bongers RM: Movement characteristics of upper extremity prostheses during basic goal-directed tasks. Clin Biomech 2010, 25:523-529.

20. Bouwsema $H$, van der Sluis CK, Bongers RM: Changes in performance over time while learning to use a myoelectric prosthesis. J Neuroeng Rehabil 2014, 11:16

21. Lee TD, Swanson LR, Hall AL: What is repeated in a repetition? Effects of practice conditions on motor skill acquisition. Phys Ther 1991, 71:150-156.

22. Bouwsema $H$, van der Sluis CK, Bongers RM: The role of order of practice in learning to handle an upper-limb prosthesis. Arch Phys Med Rehabil 2008, 89:1759-1764.

23. Kitago T, Krakauer JW: Motor learning principles for neurorehabilitation. Handb Clin Neurol 2013, 110:93-103.

24. Latash ML, Anson JG: Synergies in health and disease: relations to adaptive changes in motor coordination. Phys Ther 2006, 86:1151-1160

25. Ostlie K, Lesjo IM, Franklin RJ, Garfelt B, Skjeldal OH, Magnus P: Prosthesis use in adult acquired major upper-limb amputees: patterns of wear, prosthetic skills and the actual use of prostheses in activities of daily life. Disabil Rehabil 2012, 7:479-493.

26. Ostlie K, Lesjo IM, Franklin RJ, Garfelt B, Skjeldal OH, Magnus P: Prosthesis rejection in acquired major upper-limb amputees: a population-based survey. Disabil Rehabil 2012, 7:294-303.

27. Lake C: Effects of prosthetic training on upper-extremity prosthesis use. J Prosthet Orthot 1997, 9:3-9.

28. Lindner HY, Natterlund BS, Hermansson LM: Upper limb prosthetic outcome measures: review and content comparison based on International Classification of Functioning, Disability and Health. Prosthet Orthot Int 2010, 34:109-128.

29. Wright V: Prosthetic outcome measures for use with upper limb amputees: A systematic review of the peer-reviewed literature, 1970 to 2009. J Prosthet Orthot 2009, 21:3-63.

30. Biddiss EA, Chau TT: Upper limb prosthesis use and abandonment: a survey of the last 25 years. Prosthet Orthot Int 2007, 31:236-257.

31. Hanspal RS, Fisher K, Nieveen R: Prosthetic socket fit comfort score. Disabil Rehabil 2003, 25:1278-1280.

32. Light CM, Chappell PH, Kyberd PJ: Establishing a standardized clinical assessment tool of pathologic and prosthetic hand function: normative data, reliability, and validity. Arch Phys Med Rehabil 2002, 83:776-783.

33. Kadaba MP, Ramakrishnan HK, Wootten ME, Gainey J, Gorton G, Cochran GV: Repeatability of kinematic, kinetic, and electromyographic data in normal adult gait. J Orthop Res 1989, 7:849-860

34. Roislien J, Skare O, Opheim A, Rennie L: Evaluating the properties of the coefficient of multiple correlation (CMC) for kinematic gait data. J Biomech 2012, 45:2014-2018.

35. McGinley JL, Baker R, Wolfe R, Morris ME: The reliability of threedimensional kinematic gait measurements: a systematic review. Gait Posture 2009, 29:360-369.

36. Heyrman L, Feys H, Molenaers G, Jaspers E, Van de Walle P, Monari $D$ Aertbelien E, Desloovere K: Reliability of head and trunk kinematics during gait in children with spastic diplegia. Gait Posture 2013, 37:424-429.

37. Reid S, Elliott C, Alderson J, Lloyd D, Elliott B: Repeatability of upper limb kinematics for children with and without cerebral palsy. Gait Posture 2010, 32:10-17.

38. Fitoussi F, Diop A, Maurel N, Laassel el M, Pennecot GF: Kinematic analysis of the upper limb: a useful tool in children with cerebral palsy. J Pediatr Orthop B 2006, 15:247-256

39. Mackey AH, Walt SE, Lobb GA, Stott NS: Reliability of upper and lower limb three-dimensional kinematics in children with hemiplegia. Gait Posture 2005, 22:1-9.

40. Jaspers E, Desloovere K, Bruyninckx H, Molenaers G, Klingels K, Feys H: Review of quantitative measurements of upper limb movements in hemiplegic cerebral palsy. Gait Posture 2009, 30:395-404.

41. Jaspers E, Feys $H$, Bruyninckx H, Cutti A, Harlaar J, Molenaers G, Desloovere $\mathrm{K}$ : The reliability of upper limb kinematics in children with hemiplegic cerebral palsy. Gait Posture 2011, 33:568-575.

42. Michaelsen SM, Levin MF: Short-term effects of practice with trunk restraint on reaching movements in patients with chronic stroke: a controlled trial. Stroke 2004, 35:1914-1919. 
43. Woodbury ML, Howland DR, McGuirk TE, Davis SB, Senesac CR, Kautz S, Richards LG: Effects of trunk restraint combined with intensive task practice on poststroke upper extremity reach and function: a pilot study. Neurorehabil Neural Repair 2009, 23:78-91.

44. Michaelsen SM, Dannenbaum R, Levin MF: Task-specific training with trunk restraint on arm recovery in stroke: randomized control trial. Stroke 2006, 37:186-192.

45. Saha D, Gard S, Fatone S, Ondra S: The effect of trunk-flexed postures on balance and metabolic energy expenditure during standing. Spine 2007, 32:1605-1611.

46. Esposti R, Esposito F, Ce E, Baldissera F: Difference in the metabolic cost of postural actions during iso- and antidirectional coupled oscillations of the upper limbs in the horizontal plane. Eur J Appl Physiol 2010, 108:93-104

47. Miller LA, Lipschutz RD, Stubblefield KA, Lock BA, Huang H, Williams TW 3rd, Weir RF, Kuiken TA: Control of a six degree of freedom prosthetic arm after targeted muscle reinnervation surgery. Arch Phys Med Rehabil 2008, 89:2057-2065.

48. Stergiou N, Decker LM: Human movement variability, nonlinear dynamics, and pathology: is there a connection? Hum Mov Sci 2011, 30:869-888.

49. van de Laar B, Plass-Oude Bos D, Reuderink B, Poel M, Nijholt A: How much control is enough? Influence of unreliable input on user experience. IEEE Trans Cybern 2013, 43:1584-1592.

50. Scheirer J, Fernandez R, Klein J, Picard RW: Frustrating the user on purpose: a step toward building an affective computer. Interact Comput 2002, 14:93-118.

51. Dai B, Leigh S, Li H, Mercer VS, Yu B: The relationships between technique variability and performance in discus throwing. J Sports Sci 2013, 31:219-228.

52. Hiley MJ, Zuevsky W, Yeadon MR: Is skilled technique characterized by high or low variability? An analysis of high bar giant circles. Hum Mov Sci 2013, 32:171-180.

53. Fleisig G, Chu Y, Weber A, Andrews J: Variability in baseball pitching biomechanics among various levels of competition. Sports Biomech 2009, 8:10-21.

54. Sobuh MMD: Visuomotor behaviours during functional task performance with a myoelectric prosthesis. PhD. University of Salford, School of Health Sciences; 2012

55. Otr OV, Reinders-Messelink HA, Bongers RM, Bouwsema H, Van Der Sluis CK: The i-LIMB hand and the DMC plus hand compared: a case report. Prosthet Orthot Int 2010, 34:216-220.

56. Thies SB, Tresadern PA, Kenney LP, Smith J, Howard D, Goulermas JY, Smith C, Rigby J: Movement variability in stroke patients and controls performing two upper limb functional tasks: a new assessment methodology. J Neuroeng Rehabil 2009, 6:2.

doi:10.1186/1743-0003-11-132

Cite this article as: Major et al:: Comparison of range-of-motion and variability in upper body movements between transradial prosthesis users and able-bodied controls when executing goal-oriented tasks. Journal of NeuroEngineering and Rehabilitation 2014 11:132.

\section{Submit your next manuscript to BioMed Central and take full advantage of:}

- Convenient online submission

- Thorough peer review

- No space constraints or color figure charges

- Immediate publication on acceptance

- Inclusion in PubMed, CAS, Scopus and Google Scholar

- Research which is freely available for redistribution

Submit your manuscript at www.biomedcentral.com/submit
Ciomed Central 